\title{
Capacitive-Loaded Interstitial Antennas for Perfect Matching and Desirable SAR Distributions
}

\author{
Hee-Ran Ahn*, Senior Member, IEEE and Kwyro Lee, Senior Member, IEEE
}

\begin{abstract}
New interstitial antennas are proposed. They basically consist of coaxial cable and two types of capacitive loads. One is tipped at the end of antennas, which helps almost perfect matching possible. The others are located in the middle and needed for better specific absorption rate (SAR) distribution. To distinguish them, one at the end is called the end-capacitive load (ECL) and the others in the middle the middle-capacitive loads (MCLs). Depending on the number of the MCLs, ZMIA (zero MCL interstitial antenna), OMIA (one MCL interstitial antenna) and two MCL interstitial antenna (TMIA) are named and a matching technique based on transmission line theory is suggested. To verify the technique, the three antennas immersed in muscle phantom are designed, fabricated, measured and compared. The measured reflection coefficients of ZMIA, OMIA, and TMIA are $-28.4,-21.9$, and $-22.8 \mathrm{~dB}$, respectively, one of which, $-28.4 \mathrm{~dB}$ may be considered as the best among those reported. The compared results show that the measured ones are in good agreement with the calculated (predicted) ones. The three antennas are also measured for the SAR distributions. The measured results indicate that the TMIA has the best performance as expected and the region more than $43{ }^{\circ} \mathrm{C}$ is a rugby ball (major axis $6 \mathrm{~cm}$ and minor axis $2.9 \mathrm{~cm}$ ) with only one TMIA, which confirms that they may be used for the treatment for big-sized and deep-seated tumor or cancer.
\end{abstract}

Index Terms-Antennas matching technique and SAR distributions, hyperthermia, new types of interstitial antennas, OMIA and TMIA, transmission line equivalent circuit of the interstitial antennas, ZMIA.

\section{INTRODUCTION}

$\mathbf{T}$ HE MICROWAVE antennas are especially suitable for inducing hyperthermia of deep-seated tumors (e.g. certain brain tumors). They are inserted into medical plastic catheters embedded in target heating area, and the plastic catheters are used to avoid direct contact between antennas and body tissues. Mainly due to less expense, easy operation and short recover time, the use of the interstitial antennas has recently been on a dramatic increase, and many studies on them have been published [1]-[11]. However, none of the resulting design is directly applicable to catheter ablation of the large target (e.g. brain or liver tumors) because of not so desirable energy concentration

\footnotetext{
Manuscript received March 16, 2004; revised February 13, 2005. This work was supported by Brain Korea (BK) 21 program and MICROS Research Center, Korea Advance Institute of Science and Technology (KAIST), Korea. Asterisk indicates corresponding author.

*H. Ahn is with the Department of Electonics \& Electrical Engineering, Pohang University of Science and Technology (POSTECH), Pohang 790-784, Republic of Korea (e-mail:hrahn@ postech.ac.kr).

K. Lee is with the Division of Electrical Engineering, Department of Electrical Engineering and Computer Science (EECS), Korea Advance Institute of Science and Technology (KAIST), Taejon 305-701, Korea.

Digital Object Identifier 10.1109/TBME.2005.857640
}

in the target volume and small effective area for the treatment. Also, since some of interstitial antennas are designed with optimization [3] or not exact design method [5], antenna matching is poor [7].

In this paper, three types of interstitial antennas are proposed and designed, so that high energy concentration in the target volume can be produced for as little damage as possible to the healthy surrounding tissue. Almost perfect matching and desirable SAR distribution [12] are considered to reduce damage and two types of capacitive loads are adopted for the purpose. One is tipped at the end of the antennas, which helps almost perfect matching possible. The others are located in the middle at equal intervals and needed for better SAR distribution. To distinguish the two capacitive loads, one at the end is called the end capacitive load (ECL) and the others the middle capacitive load (MCL). Depending on the number of MCLs, zero MCL interstitial antenna (ZMIA), one MCL interstitial antenna (OMIA), and two MCL interstitial antenna (TMIA) are named, and the ZMIA is compared with conventional antennas [1]-[3], [7]. The compared results show that the ZMIA has excellent performances for the SAR distribution and smaller size.

Most of the conventional interstitial antennas have a sinusoidal current distribution with null at the end points [1]-[8], [13]-[15] and there are some with no current null at the end points [9]-[11]. However, those with current null are open-circuited at the end point and matches do not appear well in [1]-[8], [13], [14]. Those in [9]-[11] are so complicate for the fabrication and have disadvantage in terms of transversal size reduction. On the other hand, those with the ECL have no current null at the end and their input impedances can be arbitrarily changed by the ECL. Therefore, good matches can definitely be obtained. That is considered as one advantage of those with the ECL. Because of the structure of the ECL, relatively large amount of the current is concentrated around it, which induces more electron emission like a delta gap source [14] or frill generator [15]. Therefore, electric power density, directly proportional to SAR, can be concentrated around the ECL. That is another advantage. In addition, the interstitial antennas with the ECL may be realized with smaller size, which will be shown later. That may be regarded as an additional advantage with the ECL.

To design them theoretically, three transmission-line equivalent circuits are derived and a matching technique is also introduced. They base on complex propagation constants and characteristic impedances. These complex constants are derived with an approximated numerical calculation in the vicinity of the insulation [13] but good agreement is ascertained for lager target volume, radial distance greater than $10 \mathrm{~mm}$ [14]. Based on the derived transmission-line equivalent circuits, simulations 
have been carried out, which cannot be obtained from any commercial circuit simulator and the simulation results are in good agreement with the measured results. To verify the technique, the ZMIA, OMIA, and TMIA have been designed, fabricated and measured for muscle phantom, and the measured results of ZMIA, OMIA, and TMIA show $-28.4,-21.9$, and $-22.8 \mathrm{~dB}$, respectively. One of them, $-28.4 \mathrm{~dB}$, may be regarded as the best among those reported [6], [7].

For the SAR distributions, the three antennas designed for the muscle phantom have been measured and two antennas are compared with each other. The measured SAR distributions show that the TMIA is the best performance as expected. Since the temperatures above $43^{\circ} \mathrm{C}$ can be used for therapeutic purposes [17], the $43{ }^{\circ} \mathrm{C}$ isothermal line of the TMIA is investigated and the measured region greater than $43{ }^{\circ} \mathrm{C}$ is a rugby ball (major axis $6 \mathrm{~cm}$ and minor axis $2.9 \mathrm{~cm}$ ), which can be applied for a deep seated tumor or cancer.

This paper is organized as follows. The ECL and matching technique are discussed in Section II, design and frequency responses of the ZMIA, OMIA, and TMIA are explained in Section III, Section IV describes SAR distributions of the three antennas, showing the TMIA has the best performance and conclusions are in Section V.

\section{ANALYSES}

The monopole antennas are generally a quarter-wave long and classified as an image antennas. They, unlike half-wave dipole antennas that are isolated from any conductor, can utilize the reflecting properties of conductor. Due to this property, if the conductors on the antenna are properly arranged, the near field pattern may be changed. Fig. 1 shows three types of interstitial antennas where the ZMIA is described in Fig. 1(a) and the OMIA and TMIA depicted in Fig. 1(b) and (c), respectively. Each basically consists of inner conductor (radius: $a$ ), dielectric surrounding layer (relative dielectric constant: $\varepsilon_{d}$ ) and outer conductor (radius: $b$ ). Its inner conductor is extended to approximately $\lambda_{\text {eff }} / 4$ (Region 1 ) where $\lambda_{\text {eff }}$ is an effective wavelength and tipped with an ECL as displayed in Fig. 1, making a monopole antenna. The monopole antenna is insulated by a surrounding dielectric layer, its relative dielectric constant $\varepsilon_{3}$ (Region 3) with inner and outer radii, $c$ and $d$, respectively, and Region 2 is filled with air, all of which are immersed in an infinite ambient medium (Region 4). Between the aperture and ECL, one or two MCLs, just pieces of the same coaxial cable, are placed at equal intervals as shown in Fig. 1(b) and (c).

\section{A. $E C L$}

For transmitting antennas, the delta gap source [15] and frill generator [16] are commonly used as a source modeling. Although such sources do not exist in practice, they do permit surprisingly good calculations to be made. Just like them, the ECL is not a real source but most of the excited current is concentrated like a source. Fig. 2 shows the ends of the antennas where the ECL is explained in Fig. 2(a), while representative ends of conventional antennas are depicted in Fig. 2(b)-(d) [2], [3], [7].

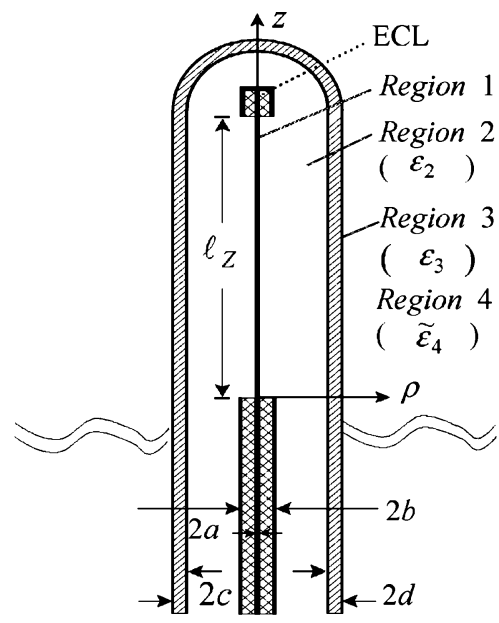

(a)

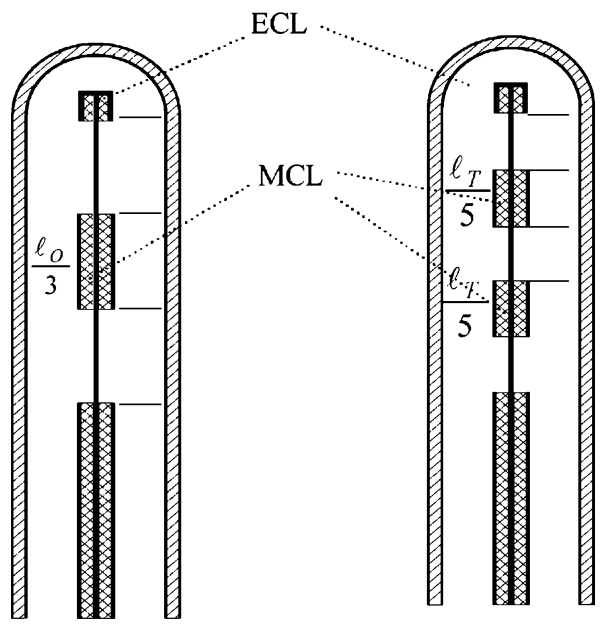

(b)

(c)

Fig. 1. New interstitial antennas. (a) Zero MCL interstitial antenna (ZMIA). (b) One MCL interstitial antenna (OMIA). (c) Two MCL interstitial antenna (TMIA).

That in Fig. 2(b) is the most popular design with the inner conductor extended, that in Fig. 2(c) forms a metal tip monopole providing increased electrical contact with tissue [7] and that in Fig. 2(d) is similar to the ECL but the inner conductor is not connected with the outer conductor. As described precisely in Fig. 2(a), the ECL is composed of a certain length of the coaxial cable and its inner conductor at the end $\left(z=\ell^{t}\right)$ is connected with the outer conductor. The outer conductor at $z=$ $\ell$ is open-circuited as shown in Fig. 2(a) where dark parts are all conductors. Therefore, when the power is fed into the antenna, the current will flow along the inner conductor of the ECL, and the opposite charge is at the same time induced on the outer conductor. Since the surface area at $z=\ell^{t}\left(\pi(b+\Delta)^{2}, \Delta\right.$ : outer conductor thickness) is much wider than cross-sectional area of the inner conductor $\left(\pi a^{2}\right)$, the current at $z=\ell^{t}$ spreads faster on the top plate. The end in Fig. 2(c) has about same effect, but the ECL has an advantage of smaller size and better SAR distribution over that in Fig. 2(c), which will be shown later. Depending on the phase of an excited signal, the induced charge on the outer conductor is determined, and Fig. 2(a) shows the case of positive current. Therefore, the approximate electricfield pattern is produced as indicated with arrows. The effect due to the induced charge also appears in Fig. 2(d). While the 


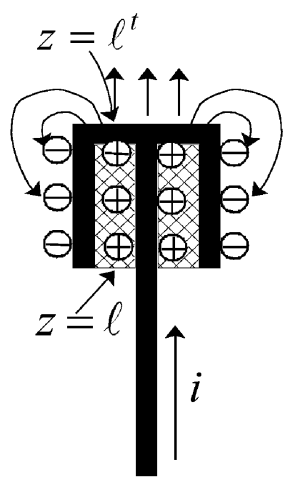

(a)



(c) (b)

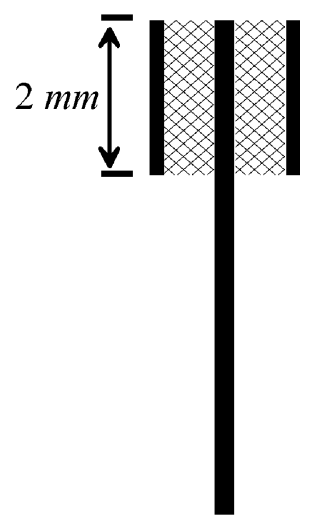

(d)
Fig. 2. Antenna ends. (a) ECL. (b) The end with inner conductor extended. (c) That with metal tip (d) that with a capacitive load.

current flows on the inner conductor surrounded with dielectric material in Fig. 2(d), the opposite charge is also induced, and electric fields from the end of the inner conductor to the outer conductor are produced. However, the cross-sectional area of the inner conductor at the end is not so wide compared to that of the ECL that the same effect may not be expected.

Therefore, the ECL can be considered as having both advantages of Fig. 2(c) and (d) with smaller size. Since the SAR is directly proportional to electric energy density [7], it has been simulated, and the ZMIA is compared with conventional antennas with the ends in Fig. 2(b)-(d). For the simulations, a semi rigid coaxial cable with inner and outer radii $0.29 \mathrm{~mm}$ and 1.4 $\mathrm{mm}$, respectively, and relative dielectric constant $\varepsilon_{d}=2.1$ (dielectric constant of coaxial cable) is utilized. Crystal glass tube $\varepsilon_{3}=5.1$ with inner and outer radii, $2.3 \mathrm{~mm}$ and $4.2 \mathrm{~mm}$ are used as a microwave catheter. The antennas are designed for good matching. The length of the ECL is $2 \mathrm{~mm}$ and those of the two others in Fig. 2(c) and (d) are also $2 \mathrm{~mm}$. They are immersed in air, and air also fills the gap between the antennas and crystal glass tubes. The crystal glass tube and the excited power are the same in each case. How to design them will be discussed later. The four antennas have been simulated by CST (computer simulation technology, 3D electro-magnetic field simulator) Microwave Studio, version 4.2 and the simulated electric energy densities are plotted in Fig. 3 where that of the ZMIA is in Fig. 3(a), and Fig. 3(b)-(d) are those of conventional ones with the ends in Fig. 2(b)-(d), respectively. As shown in Fig. 3, the length of ZMIA is $12.7 \mathrm{~mm}$, those of others are $20.2 \mathrm{~mm}, 14$ $\mathrm{mm}$ and $18 \mathrm{~mm}$ in Fig. 3(b)-(d), respectively. The ZMIA may
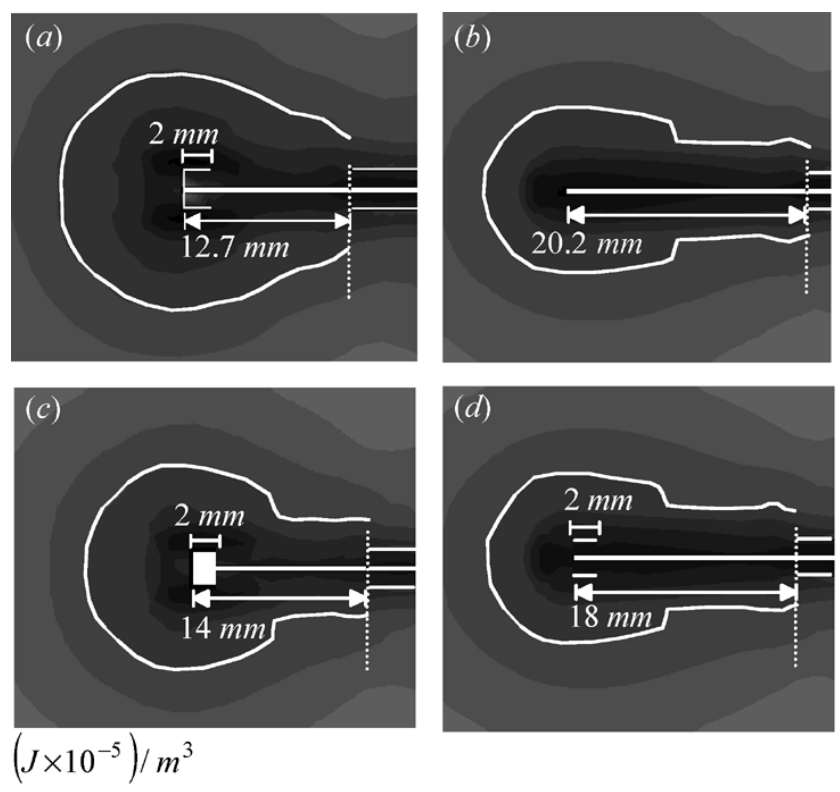

$\begin{array}{llll}139 & 24.8 & 2.46 & 0.234\end{array}$

\section{0}

$\begin{array}{llll}-0.234 & -2.46 & -24.8 & -139\end{array}$

Fig. 3. Simulated electric energy density. (a) ZMIA. (b) The antenna with the inner conductor extended in Fig. 2(b). (c) That tipped with metal in Fig. 2(c). (d) That tipped with capacitive load in Fig. 2(d).

be designed with the shortest length, which is also an advantage of antenna with the ECL. In Fig. 3, the solid lines express same electric energy density and they are compared in the same scale. The contour in Fig. 3(b) is very similar to that of Fig. 3(d), but the antenna length of Fig. 3(d) is shorter than that in Fig. 3(b). The ZMIA is compared with others and the results are plotted in Fig. 4 where the solid lines are the contours of same electric energy density. In Fig. 4(a), the ZMIA is compared with that with no tip in Fig. 2(b). In Fig. 4(b) and (c), it is also compared with those with a metal tip and a dielectric tip surrounded with outer conductor in Fig. 2(c) and (d), respectively. From the simulation results in Fig. 4, the contour of the ZMIA is largest and its shape is very similar to tumor or cancer configuration. It is also very useful aspect for the hyperthermia application.

\section{B. Antenna Matching}

In the case these interstitial antennas are utilized for human body, antenna matching is important for the field concentration and protecting a microwave radiator (source) from damage during operating. When these interstitial antennas in Fig. 1 are placed in dissipative media, they may be treated as sections of lossy transmission lines with generalized propagation constants that reflect the losses due to radiation from the antennas to the ambient medium. The propagation constants of the dielectric layers in the Regions 2, 3, and 4 in Fig. 1 are $k_{2}=\omega \sqrt{\mu_{0} \varepsilon_{0} \varepsilon_{2}}$, $k_{3}=\omega \sqrt{\mu_{0} \varepsilon_{0} \varepsilon_{3}}$ and $k_{4}=\beta_{4}+i \alpha_{4}=\omega \sqrt{\mu_{0} \varepsilon_{0} \tilde{\varepsilon}_{4}}, \tilde{\varepsilon}_{4}=$ $\varepsilon_{4}+i \sigma_{4} / \omega$, respectively where $\varepsilon_{2}$ and $\varepsilon_{3}$ are assumed to be real and dielectric constants for Regions 2 and 3, respectively, and $\tilde{\varepsilon}_{4}$ complex since the dielectrics actually used in the Regions 2 and 3 are highly nonconducting, while that of the ambient medium of the Region 4 conducting.

Fig. 5 shows the equivalent circuits of the ZMIA, OMIA, and TMIA when they are immersed in the lossy medium. The ZMIA is depicted in Fig. 5(a), the OMIA and TMIA in Fig. 5(b) and 


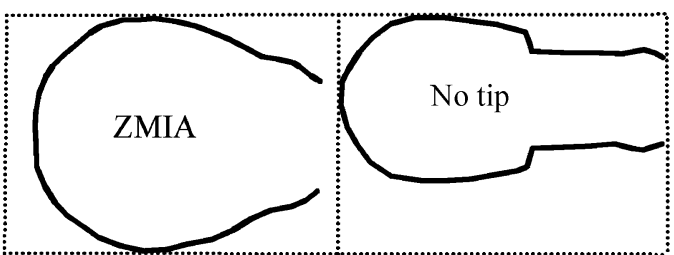

(a)

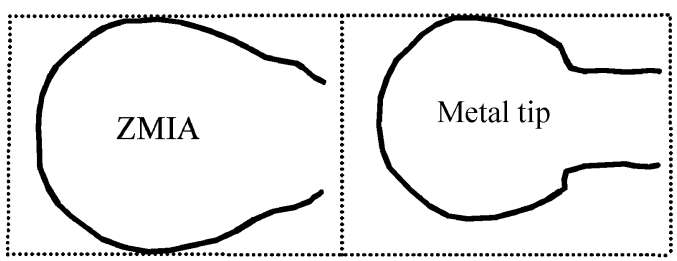

(b)

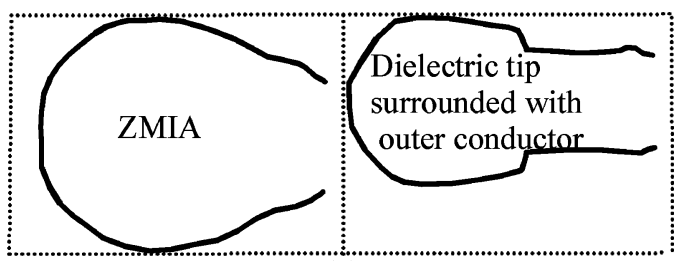

(c)

Fig. 4. Compared electric energy densities. (a) ZMIA and an antenna with no tip. (b) ZMIA and an antenna with a metal tip. (c) ZMIA and an antenna with a dielectric tip surrounded with outer conductor in Fig. 2(d).

(c), respectively where the antenna lengths are $l_{Z}, l_{O}$, and $l_{T}$ for the ZMIA, OMIA, and TMIA, respectively. The input impedances at $z=0$ are $Z_{\text {in }-Z}, Z_{\text {in }-O}$, and $Z_{\text {in }-T}$ for the ZMIA, OMIA, and TMIA, respectively, and that of the ECL $Z_{L}$. For a dielectric with two layers, the propagation constant $k_{c}(r)$ and its characteristic impedance $Z_{c}(r)$ [13] are derived as

$$
\begin{aligned}
k_{c}(r)= & k_{2 e}(r)\left[\frac{\ln \left(\frac{d}{r}\right)+F}{\ln \left(\frac{d}{r}\right)+n_{24}^{2} F}\right]^{\frac{1}{2}} \\
Z_{c}(r)= & \left(\frac{\omega \mu_{0} k_{c}(r)}{2 \pi k_{2}^{2}}\right) \\
& \times\left[\ln \left(\frac{d}{r}\right)+n_{23}^{2} \ln \left(\frac{d}{c}\right)+n_{24}^{2} F\right] \text { where } \\
k_{2 e}(r)= & k_{2}\left[\frac{\ln \left(\frac{d}{r}\right)}{\ln \left(\frac{c}{r}\right)+n_{23}^{2} \ln \left(\frac{d}{c}\right)}\right]^{\frac{1}{2}} \\
n_{23}^{2}= & \frac{k_{2}^{2}}{k_{3}^{2}}, \quad n_{24}^{2}=\frac{k_{2}^{2}}{k_{4}^{2}} \quad \text { and } \\
F= & \frac{H_{0}^{(1)}\left(k_{4} d\right)}{\left(k_{4} d H_{1}^{1}\left(k_{4} d\right)\right)} \\
& \text { with } H: \text { Hankel function. }
\end{aligned}
$$

(1c)

The $k_{c}(r)$ and $Z_{c}(r)$ are valid only when the propagation constant of the ambient medium is large compared to that of insulating sheath and the cross section of the antenna is electrically small [13]. That is

$$
\begin{aligned}
& \left|\frac{k_{4}}{k_{2}}\right|^{2} \gg 1 ; \quad\left|\frac{k_{4}}{k_{3}}\right|^{2} \gg 1 ; \\
& \left(k_{2} c\right)^{2} \ll 1 ; \quad\left(k_{3} d\right)^{2} \ll 1
\end{aligned}
$$

The $k_{c}(r)$ and $Z_{c}(r)$ are dependent on the radius and they are $k_{c}(a)$ and $Z_{c}(a)$ in the case of the ZMIA as shown in Fig. 5. (a)

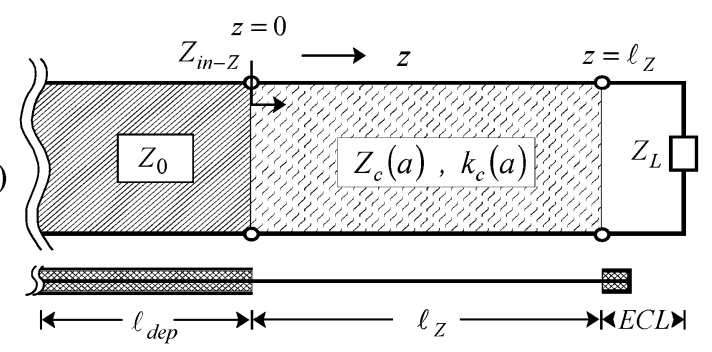

(b)

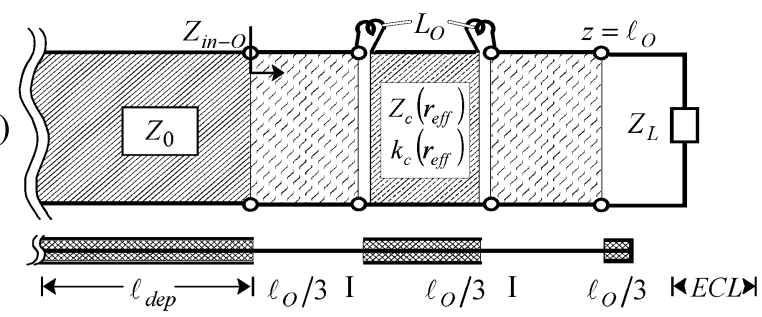

(c)

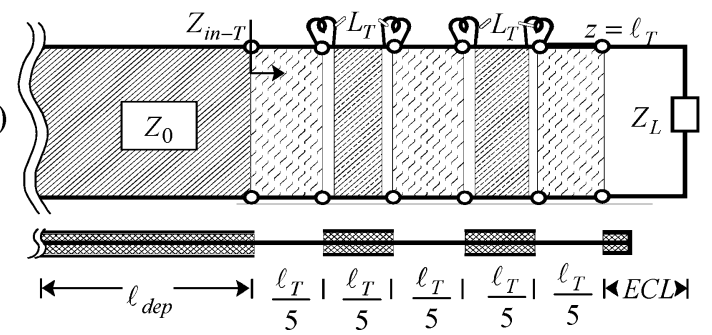

Fig. 5. Transmission line equivalent circuits of the interstitial antennas. (a) ZMIA, (b) OMIA, (c) TMIA.

Therefore, the input impedance $Z_{\text {in }-Z}$ is calculated using a simple transmission line theory and expressed by

$$
Z_{\text {in }-Z}=Z_{c}(a) \frac{Z_{L}+j Z_{c}(a) \tan \left[k_{c}(a) \ell_{z}\right]}{Z_{c}(a)+j Z_{L} \tan \left[k_{c}(a) \ell_{z}\right]} .
$$

In the equivalent circuit of the OMIA in Fig. 5(b), the inductance appears. It is mainly due to charge movement from the ECL to the aperture. Therefore, the outer conductor of the MCL at $z=\ell_{O} / 3$ to $z=2 \ell_{O} / 3$ helps electric field around the center of the OMIA more concentrated and it plays role of a kind of bridge connected between the ECL and aperture. In the TMIA in Fig. 1(c) and (5c), since two MCLs are located between the aperture and ECL, it will be shown later that more field concentration is expected than in OMIA. The $k_{c}(r)$ and $Z_{c}(r)$ of the transmission line at $z=2 \ell_{O} / 3$ to $z=\ell_{O}$ in Fig. 5(b) are $k_{c}(a)$ and $Z_{c}(a)$, respectively. So, the input impedance $Z_{\text {in-2 }} \ell_{0} / 3$ at $z=2 \ell_{O} / 3$ is expressed by

$$
Z_{\text {in- } \frac{2 \ell_{O}}{3}}=Z_{c}(a) \frac{Z_{L}+j Z_{c}(a) \tan \left[k_{c}(a) \frac{\ell_{O}}{3}\right]}{Z_{c}(a)+j Z_{L} \tan \left[k_{c}(a) \frac{\ell_{O}}{3}\right]}+j X_{O}
$$

where $X_{O}$ is due to the inductance and very small value of 0.2 . The propagation constant and characteristic impedance of the transmission line located at $z=\ell_{O} / 3$ to $z=2 \ell_{O} / 3$ in OMIA must be different from $k_{c}(a)$ and $Z_{c}(a)$ of ZMIA in Fig. 5(a). The $k_{c}(r)$ and $Z_{c}(r)$ in (1) are dependent on the radius and if let the effective radius of OMIA at $z=\ell_{O} / 3$ to $z=2 \ell_{O} / 3 r_{\text {eff }}$, the input impedance $Z_{\mathrm{in}-\ell_{O} / 3}$ at $z=\ell_{O} / 3$ is given by

$$
\begin{aligned}
Z_{\mathrm{in}-\frac{\ell_{O}}{3}} & =Z_{c}\left(r_{\mathrm{eff}}\right) \\
& \times \frac{Z_{\mathrm{in}-\frac{2 \ell_{O}}{3}}+j Z_{c}\left(r_{\mathrm{eff}}\right) \tan \left[k_{c}\left(r_{\mathrm{eff}}\right) \frac{\ell_{O}}{3}\right]}{Z_{c}\left(r_{\mathrm{eff}}\right)+j Z_{\mathrm{in}-\frac{2 \ell_{O}}{3}} \tan \left[k_{c}\left(r_{\mathrm{eff}}\right) \frac{\ell_{O}}{3}\right]}+j X_{O} .
\end{aligned}
$$


(a)



(b)

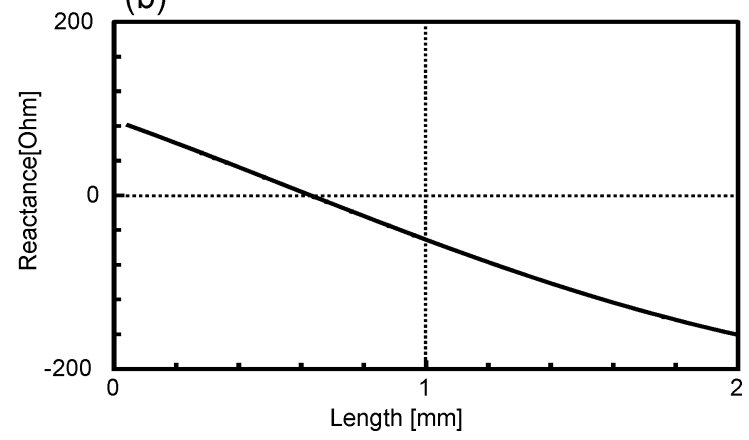

Fig. 6. Simulated impedances of the ECL. (a) Resistances; (b) reactances.

The $Z_{\mathrm{in}-O}$ is composed of $Z_{\mathrm{in}-\ell_{O} / 3}$ and $Z_{\mathrm{in}-2 \ell_{O} / 3}$, and finally results in

$$
Z_{\mathrm{in}-O}=Z_{c}(a) \frac{Z_{\mathrm{in}-\frac{\ell_{O}}{3}}+j Z_{c}(a) \tan \left[k_{c}(a) \frac{\ell_{O}}{3}\right]}{Z_{c}(a)+j Z_{\mathrm{in}-\frac{\ell_{O}}{3}} \tan \left[k_{c}(a) \frac{\ell_{O}}{3}\right]} .
$$

As expressed in (3)-(6), each input impedance consists of a characteristic impedance $Z_{c}$, a propagation constant $k_{c}$, a length of transmission-line section $\ell$ and a termination load impedance $Z_{L}$. If a function expressing the input impedance is defined as $\operatorname{Load}\left(Z_{c}, k_{c}, \ell, Z_{L}\right)$, the input impedance $Z_{\mathrm{in}-N}$ of interstitial antennas with arbitrary number of MCLs may be derived in sequence as follows:

$$
\begin{aligned}
Z_{\mathrm{in}-N}= & \operatorname{Load}\left(Z_{c}(a), k_{c}(a), \Delta \ell, Z_{\mathrm{in}-\frac{\ell}{(2 N+1)}}\right) \\
Z_{\mathrm{in}-\frac{\ell}{(2 N+1)}=} & \operatorname{Load}\left(Z_{c}\left(r_{\mathrm{eff}}\right), k_{c}\left(r_{\mathrm{eff}}\right), \Delta \ell, Z_{\mathrm{in}-\frac{2 \ell}{(2 N+1)}}\right) \\
& +j X_{N} \\
\bullet & \\
Z_{\mathrm{in}-\frac{(2 N-1) \ell}{(2 N+1)}=} & \operatorname{Load}\left(Z_{c}\left(r_{\mathrm{eff}}\right), k_{c}\left(r_{\mathrm{eff}}\right), \Delta \ell, Z_{\mathrm{in}-\frac{2 N \ell}{(2 N+1)}}\right) \\
& +j X_{N} \\
Z_{\mathrm{in}-\frac{2 N \ell}{(2 N+1)}} & \operatorname{Load}\left(Z_{c}(a), k_{c}(a), \Delta \ell, Z_{L}\right)+j X_{N} .
\end{aligned}
$$

where $N$ is the number of MCL, $Z_{\mathrm{in}-P}$ is an input impedance at $z=P \leq \ell, \Delta \ell=\ell /(2 N+1)$ and $j X_{N}=j 0.2$ at $2.45 \mathrm{GHz}$.

\section{DESIGN AND FREQUENCY RESPONSES}

For the design of the antennas, the input impedance of the ECL is needed. Fig. 6 shows calculated input impedances of the ECL depending on the length of $\ell_{S}$ where $\ell_{S}=\ell_{t}-\ell$ in Fig. 2(a). The $\ell_{S}$ is varied from $0 \mathrm{~mm}$ to $2 \mathrm{~mm}$ as shown in Fig. 6. For the calculation of the input impedance of ECL,
TABLE I

DATA FOR THE ANTENNAS AT $2.45 \mathrm{GHz}\left(r_{\text {eff }}=1.518 \times 10^{-4} \mathrm{~m}\right.$, $\left.Z_{L}=419.8-j 163.8\right)$

\begin{tabular}{c|c|c|c}
\hline $2 a$ & $2 b$ & $2 c$ & $2 d$ \\
\hline $0.29 \mathrm{~mm}$ & $1.19 \mathrm{~mm}$ & $2.3 \mathrm{~mm}$ & $4.2 \mathrm{~mm}$ \\
\hline$\varepsilon_{d}$ & $\varepsilon_{2}$ & $\varepsilon_{3}$ & $\widetilde{\varepsilon}_{4}$ \\
\hline 2.1 & 1 & 5.1 & $52.7+j 13.3$ \\
\hline$k_{c}(a)$ & $Z_{c}(a)$ & $k_{c}\left(r_{e f f}\right)$ & $Z_{c}\left(r_{e f f}\right)$ \\
{$\left[\mathrm{m}^{-1}\right]$} & {$[\Omega]$} & {$\left[\mathrm{m}^{-1}\right]$} & {$[\Omega]$} \\
\hline $66.9+i 9.6$ & $219.8+i 32.8$ & $67.26+i 9.8$ & $217.2+i 32.8$ \\
\hline
\end{tabular}

a prototype of ZMIA with $17 \mathrm{~mm}$ is fabricated and measured. The inner and outer radii of the coaxial cable are $2 a=0.29 \mathrm{~mm}$, $2 b=1.19 \mathrm{~mm}$, respectively, and its relative dielectric constant $\varepsilon_{d}=2.1$. Those of the crystal glass tube are $2 c=2.3 \mathrm{~mm}$, $2 b=1.19 \mathrm{~mm}$, respectively and $\varepsilon_{3}=5.1$. For the measurement, the ZMIA in Fig. 1(a) is immersed in a $10 \mathrm{~cm} \times 10 \mathrm{~cm} \times$ $10 \mathrm{~cm}$ muscle phantom (complex relative dielectric constant $\left.\tilde{\varepsilon}_{4}=52.7+i 13.3\right)$. In this case, the measured return loss at $2.45 \mathrm{GHz}$ is $-12.27 \mathrm{~dB}$ and the input impedance of $Z_{\text {in }-Z}$ may be calculated using $\left(Z_{\text {in }-Z}-50\right) /\left(Z_{\text {in }-Z}+50\right)$. With $Z_{C}(a)$, $k_{C}(a)$ in (1), the $Z_{L}$ can be calculated using (3). The values of $Z_{L}$ are those with $\ell_{s}=2 \mathrm{~mm}$ in Fig. 6 . Therefore, those with $\ell_{s}=0$ are calculated with $Z_{C}(b)$ and $k_{C}(b)$ in (1) and the two graphs in Fig. 6 are obtained with Mathematica (a commercial program). The resistances are plotted in Fig. 6(a), whereas the reactances in Fig. 6(b). Based on the $Z_{L}$, three antennas, ZMIA, OMIA, and TMIA with the same dimensions for Fig. 6 have been designed and fabricated.

In the case, the propagation constants of the three materials and the equivalent single-layer dielectric at $2.54 \mathrm{GHz}$ are $k_{2}=51.3 \mathrm{~m}^{-1}, k_{3}=115.9 \mathrm{~m}^{-1}$, $k_{4}=375.7+46.7 \mathrm{~m}^{-1}$ and $k_{2 e}=56.7 \mathrm{~m}^{-1}$ from (1). With $\left|k_{4} / k_{2}\right|^{2}=54.3,\left|k_{4} / k_{3}\right|^{2}=10.7,\left(k_{2} c\right)^{2}=3.48 \times 10^{-3}$ and $\left(k_{3} d\right)^{2}=5.9 \times 10^{-2}$, the condition (2) is well satisfied and their corresponding propagation constant and characteristic impedance of the transmission line of the ZMIA are $k_{c}(a)=66.9+i 9.6 \mathrm{~m}^{-1}$ and $Z_{c}(a)=219.8+i 32.84 \Omega$. All the data needed for the design are written in Table I where $k_{c}\left(r_{\text {eff }}\right)$ and $Z_{c}\left(r_{\text {eff }}\right)$ are effective propagation constant and characteristic impedance, respectively and expressed as darker meshed transmission line sections of the OMIA and TMIA in Fig. 5. How to find out them will be explained later. When the length of ECL $\ell_{S}=2 \mathrm{~mm}$, the input impedance of the ECL is obtained as $Z_{L}=419.8-j 163.8$ from Fig. 6 , and the $Z_{\text {in }-Z}$ is the function of $l_{Z}$ with the given values of $k_{c}(a), Z_{c}(a)$ and $Z_{L}$. With one $Z_{\text {in }-Z}$ given, the length of ZMIA may be solved as the solution of (3), or $\ell_{Z}=18.75 \mathrm{~mm}$. For the determination of $l_{O}$ in Fig. 5(b), $\ell_{O}$ and $r_{\text {eff }}$ are unknown variables in (4)-(6), which must ultimately be solved for the $Z_{\text {in- } O}$ in (6). Varying the $r_{\text {eff }}$ and $l_{O}$ at the same time in (6), the minimum reflection coefficient may be found out. The $r_{\text {eff }}$ and $\ell_{O}$ are calculated as $151.8 \mathrm{um}$ and $18.8 \mathrm{~mm}$, respectively with the minimum value of the reflection coefficient. Based on the known value of $r_{\mathrm{eff}}$, $\ell_{T}=18.9 \mathrm{~mm}$ can also be obtained for $N=2$ in (7). 


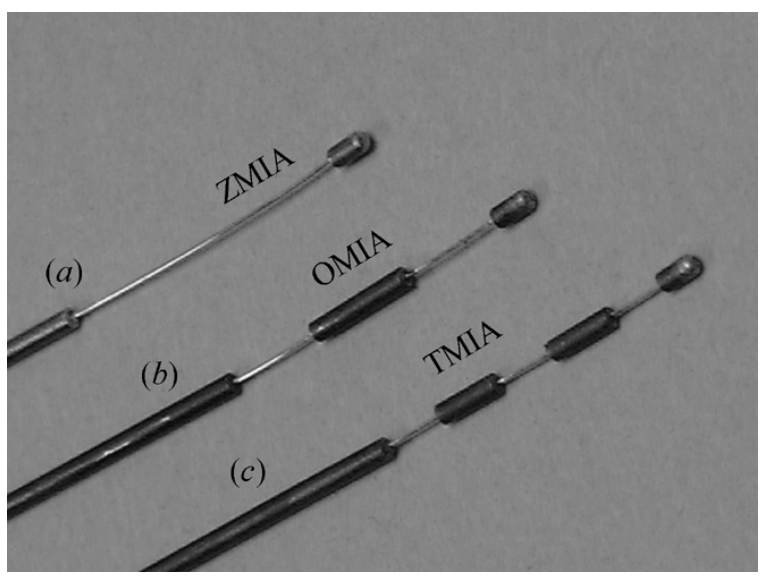

Fig. 7. The fabricated antennas without crystal glass tubes. (a) ZMIA, (b) OMIA, (c) TMIA.

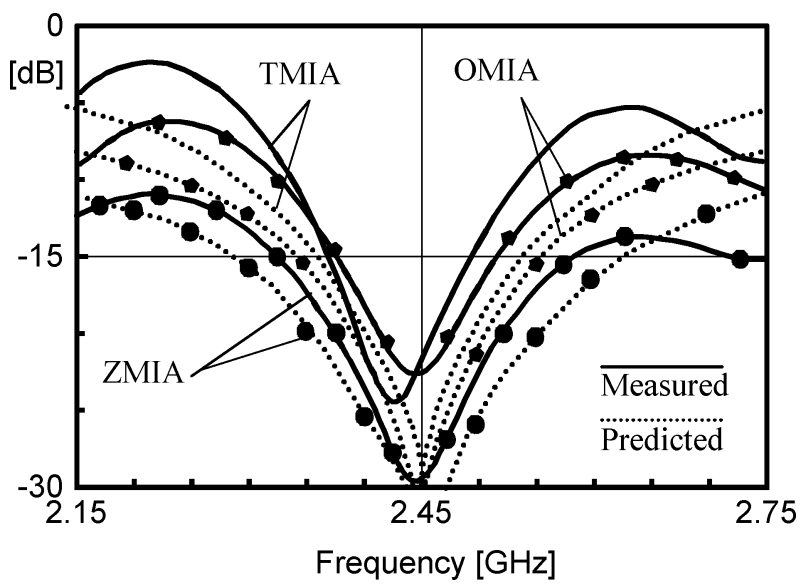

Fig. 8. Measured and calculated frequency responses: solid lines measured and dotted lines calculated.

The three antennas have been fabricated with the calculated lengths. Their photo is displayed without the crystal glass tube for the better understanding in Fig. 7 where the ZMIA is shown in Fig. 7(a), whereas the OMIA and TMIA in Fig. 7(b) and (c), respectively. Since $k_{c}(r), Z_{c}(r), k_{c}\left(r_{\text {eff }}\right)$, and $Z_{c}\left(r_{\text {eff }}\right)$ are dependent on frequencies, the frequency responses have been calculated on the basis of (1)-(7) and the calculated results are compared with measured ones in Fig. 8. For the measurements, a $10 \mathrm{~cm} \times 10 \mathrm{~cm} \times 10 \mathrm{~cm}$ muscle phantom is used and the inserted sleeve length $\ell_{d e p}$ in Fig. 5 is about $4 \mathrm{~cm}$. As far as a good matching appears, the $\ell_{\text {dep }}$ is of no importance in terms of matching problem, but the guided wave length from the antenna aperture to a connector, e.g. SMA connector must be multiple of $\lambda_{g} / 2$ where $\lambda_{g}$ is a guided wave length and with even multiple of guided wave length, the better.

The measured results are also compared in Table II. The measured bandwidths where reflection coefficients are less than -15 $\mathrm{dB}$ are $2.33 \leq f \leq 2.578 \mathrm{GHz}$ in ZMIA, $2.37 \leq f \leq$ $2.51 \mathrm{GHz}$ in OMIA, and $2.37 \leq f \leq 2.47 \mathrm{GHz}$ in TMIA and the measured reflected coefficients of the ZMIA, OMIA, and TMIA at $2.45 \mathrm{GHz}$ are $-28.4,-21.9$, and $-22.8 \mathrm{~dB}$, respectively. The calculated reflection coefficients show perfect performances, -44.5 to $-30 \mathrm{~dB}$ at $2.45 \mathrm{GHz}$. The complex relative dielectric constant of the ambient medium is generally frequency
TABLE II

COMPARISON BETWEen MEASURED AND SiMULATED REFLECTION COEFFICIENTS

\begin{tabular}{|c|c|c|}
\hline \multicolumn{3}{|c|}{$|\Gamma| \leq-15 d B$} \\
\hline & Measured[GHz] & Calculated $[\mathrm{GHz}]$ \\
\hline ZMIA & $2.33 \leq f \leq 2.578$ & $2.20 \leq f \leq 2.7$ \\
\hline OMIA & $2.37 \leq f \leq 2.51$ & $2.34 \leq f \leq 2.54$ \\
\hline TMIA & $2.37 \leq f \leq 2.47$ & $2.38 \leq f \leq 2.51$ \\
\hline \multicolumn{3}{|c|}{$|\Gamma|$ at $2.45 \mathrm{GHz}$} \\
\hline ZMIA & $-28.4 \mathrm{~dB}$ & $\leq-30 \mathrm{~dB}$ \\
\hline OMIA & $-21.9 \mathrm{~dB}$ & $\leq-30 \mathrm{~dB}$ \\
\hline TMIA & $\begin{array}{r}-22.8 \mathrm{~dB},-24.4 \mathrm{~dB} \\
(\text { at } 2.43 \mathrm{GHz})\end{array}$ & $\leq-30 \mathrm{~dB}$ \\
\hline
\end{tabular}

dependent and the relative dielectric constants of all the regions have errors but the facts are not considered for the modeling of the antennas in Fig. 5. That may be one of reasons of the deviations between measured and calculated ones. Except that point, the measured matching properties at $2.45 \mathrm{GHz}$ agree well with the calculated ones, and $-28.4 \mathrm{~dB}$ seems to be the best among this type of conventional interstitial antennas [6], [7]. The two antennas, ZMIA and OMIA are resonant at the $2.45 \mathrm{GHz}$ but the resonant frequency in the TMIA occurs at $2.415 \mathrm{GHz}$.

\section{SAR DistRIBUTION AND MEASUREMENTS}

One of the main objectives of applying microwaves for cancer or tumor treatment is to develop possibilities of delivering heat to deep-seated tumors without overheating the surrounding healthy tissue. For this purpose, high power generators and good matched antennas are especially necessary for energy concentration and desirable SAR distributions. Fig. 9 shows a microwave power generator which consists of a $2.45-\mathrm{GHz}$ oscillator, a programmable attenuator, a linear power amplifier, an isolator and a bi-directional coupler. The power generated at $2.45 \mathrm{GHz}$ by the oscillator is modulated by the programmable attenuator and amplified by the linear amplifier. Since the power amplifiers are generally designed for the maximum output power with a power matching technique, the output matching is not always desirable. Thus, an isolator is needed. The output power through the isolator needs to be examined whether it is available for the excitation to the antennas. For the purpose, the bi-directional coupler is adopted, by which the forward and reflected powers referenced to the output power of the linear amplifier are detected and displayed on the monitor. From the linear power amplifier, the forward and reflected powers are monitored, and the monitored data reflects the programming attenuator which controls the level of the input of the linear power amplifier, and if the temperature of the linear power amp is high, a cooling system operates. When the reflected power detected from the bi-directional coupler exceeds more than a right level, a tuner, by which it can automatically be reduced, was needed for the conventional coaxial type of interstitial antennas. However, the tuner is not necessary for the three antennas, ZMIA, OMIA, and TMIA 


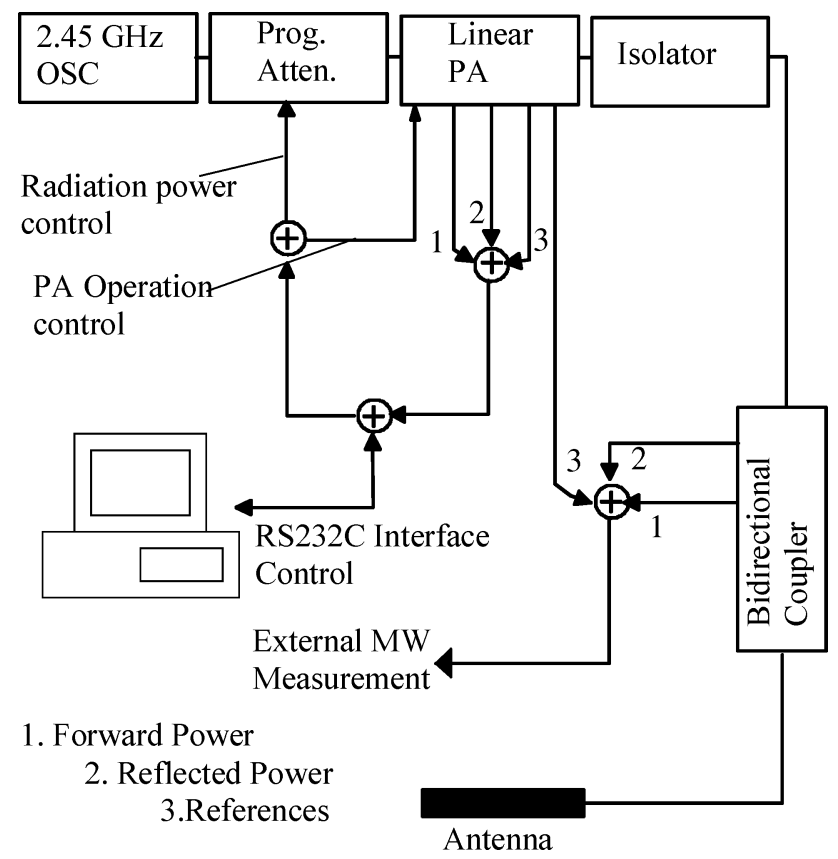

Fig. 9. Block diagram of microwave power generator used for SAR distributions of the antennas.


Fig. 10. Measured SAR distributions. (a) SAR distribution pattern of a cross section at $z=10 \mathrm{~mm}$ of OMIA (b) comparison between TMIA and OMIA, (c) comparison between OMIA and ZMIA (d) comparison between TMIA and ZMIA.

with the reflection coefficients less than $-21 \mathrm{~dB}$, which brings advantages of saving size and cost.

For measuring SAR distributions, four thermometer fiber optic sensors are attached at four different points on the antennas, and if any of four reaches at $100^{\circ} \mathrm{C}$, the power supplied by the generator is automatically stopped. Fig. 10 shows the
TABLE III

MEASURED SAR DISTRIBUTIONS

\begin{tabular}{l|l|l}
\hline Fig. 10(b) & TMIA & OMIA \\
\hline$|z|$ & $59.7 \mathrm{~mm}$ & $63.3 \mathrm{~mm}$ \\
\hline$|\rho|$ & $28.74 \mathrm{~mm}$ & $25.59 \mathrm{~mm}$ \\
\hline Fig. 10(c) & OMIA & ZMIA \\
\hline$|z|$ & $60.02 \mathrm{~mm}$ & $68.99 \mathrm{~mm}$ \\
\hline$|\rho|$ & $27.07 \mathrm{~mm}$ & $24.20 \mathrm{~mm}$ \\
\hline Fig. 10(d) & TMIA & ZMIA \\
\hline$|z|$ & $57.84 \mathrm{~mm}$ & $57.94 \mathrm{~mm}$ \\
\hline$|\rho|$ & $25.23 \mathrm{~mm}$ & $21.98 \mathrm{~mm}$ \\
\hline
\end{tabular}

measured SAR distributions pictured by IRCON (Inspect IR 500 PS) digital camera, serial number SS-7. Fig. 10(a) shows a cross-sectional temperature distribution on $z=10 \mathrm{~cm}$ plane of the OMIA in Fig. 5(b), and Fig. 10(b)-(d) shows the compared ones on $\Phi=0^{\circ}$ plane in cylindrical coordinates. The size of the muscle phantom used for the OMIA in Fig. 10(a) is $5 \mathrm{~cm} \times 5 \mathrm{~cm} \times 5 \mathrm{~cm}$ and that for Fig. 10(b)-(d) $10 \mathrm{~cm} \times 10 \mathrm{~cm} \times 10 \mathrm{~cm}$. The distance between two antennas is $5 \mathrm{~cm}$. Since the power delivered to the antenna is not same whenever measured, two-by-two are compared and the power applied to each one is from a power divider connected with the bi-directional coupler. In Fig. 10, the dotted lines are $43{ }^{\circ} \mathrm{C}$ isothermal lines. Fig. 10(a) shows that the diameter of a circle satisfied with a $43{ }^{\circ} \mathrm{C}$ isothermal line is $30 \mathrm{~mm}$ and the compared data in Fig. 10(b)-(d) are written in Table III where $|\rho|$ is maximum diameter of a circle which a $43{ }^{\circ} \mathrm{C}$ isothermal line meets and $|z|$ is maximum distance of $z$-axis cut by it. In Fig. 10(b), the TMIA is compared with the OMIA. The $|z|$ of the TMIA is $59.7 \mathrm{~mm}$ and That of OMIA $63.3 \mathrm{~mm}$. The $|\rho|$ of the TMIA is $28.74 \mathrm{~mm}$ and That of OMIA $25.59 \mathrm{~mm}$. If the $|z|$ is shorter, the better and if the $|\rho|$ is longer, the better, because of the characteristics of the tumor or cancer tissue. Thus, the TMIA has better properties in Fig. 10(b). In the same principle, the OMIA is better than the ZMIA in Fig. 10(c), and the TMIA better than the ZMIA in Fig. 10(d), which results in that the TMIA has the best performances in SAR distribution as expected before.

\section{CONCLUSION}

In this paper, three new interstitial antennas are proposed. They mainly consist of a coaxial cable, ECL, and MCLs. The ECL is useful for good impedance matching, smaller size and energy concentration around the antennas and the MCL for determining the SAR distributions more desirable. Depending on the number of the capacitive loads, the ZMIA, OMIA, and TMIA have been named and a matching technique is suggested, so that they can be designed exactly. The measured reflection coefficients of the ZMIA, OMIA, and TMIA are less than -21 $\mathrm{dB}$ at $2.45 \mathrm{GHz}$, and $-28.4 \mathrm{~dB}$ of ZMIA may be regarded as the best among those reported. The three antennas designed have been tested for the SAR distributions, and the TMIA shows the best performance as expected. If a good combination between 
number and place of the capacitive loads is made, the more desirable SAR distribution pattern may be obtained. From the measured SAR distribution of the TMIA, the region greater than $43{ }^{\circ} \mathrm{C}$ forms a rugby ball shape (major axis $6 \mathrm{~cm}$ and minor axis $2.87 \mathrm{~cm}$ ), by which a deep seated and large-sized tumor can be treated with only one antenna. Using the matching technique suggested and the input impedance of the ECL, any transversal size of the interstitial antennas can be designed for big-sized and deep-seated tumor to reduce damage to healthy surrounding tissue.

\section{ACKNOWLEDGMENT}

The authors would like to thank Prof. T. Itoh at the University of California, Los Angeles and the reviewers of this manuscript for their efforts in improving its quality.

\section{REFERENCES}

[1] Y. Zang, N. V. Dubal, R. T-Hambleton, and W. T. Joines, "The determination of the electromagnetic field and SAR pattern of an interstitial applicator in a dissipative dielectric medium," IEEE Trans. Microw. Theory Tech., vol. 36, no. 10, pp. 1438-1444, Oct. 1988.

[2] A. M. Tumeh and M. F. Iskander, "Performance comparison of available interstitial antennas for microwave hyperthermia," IEEE Trans. Microw. Theory Tech., vol. 37, no. 7, pp. 1126-1133, Jul. 1989.

[3] M. F. Iskander and A. M. Tumeh, "Design optimization of interstitial antennas," IEEE Trans. Biomed. Eng., vol. 36, no. 2, pp. 238-246, Feb. 1989.

[4] T. P. Ryan, "Techniques for heating brain tumors with implanted microwave antennas," in IEEE MTT-S Dig., 1991, pp. 791-794.

[5] W. Hürter, F. Reinbold, and W. J. Lorenz, "A dipole antenna for interstitial microwave hyperthermia," IEEE Trans. Microw. Theory Tech., vol. 39, no. 6, pp. 1048-1053, Jun. 1991.

[6] J.-C. Camart, J.-J. Fabre, B. Prevost, J. Pribetich, and M. Chive, "Coaxial antenna array for $915 \mathrm{MHz}$ interstitial hyperthermia: design and modelization-power deposition and heating pattern-phased array," IEEE Trans. Microw. Theory Tech., vol. 40, no. 12, pp. 2243-2250, Dec. 1992.

[7] S. Labont, A. Blais, S. R. Legault, H. O. Ali, and L. Roy, "Monopole antennas for microwave catheter ablation," IEEE Trans. Microw. Theory Tech., vol. 44, no. 10, pp. 1832-1840, Oct. 1996

[8] J. C. Lin and Y. J. Wang, "Interstitial microwave antennas for thermal therapy," Int. J. Hypertherm., vol. 3, pp. 37-47, 1987.

[9] - "The cap-slot catheter antenna for microwave ablation therapy," IEEE Trans. Biomed. Eng., vol. 43, no. 6, pp. 657-659, Jun. 1996.

[10] S. Pisa, S. Cavagnaro, P. Bernardi, and J. C. Lin, "A 915-MHz antenna for microwave thermal ablation treatment: physical design computer modeling and experimental measurement," IEEE Trans. Biomed. Eng., vol. 48, no. 5, pp. 599-601, May 2001.

[11] J. C. Lin, "Catheter microwave ablation therapy for cardiac arrhythmias," Bioelectromagnetics, suppl. 4, vol. 20, pp. 120-132, 1999.

[12] H.-R. Ahn and K. Lee, "Interstitial antennas tipped with reactive load," IEEE Microw. Compon. Lett., vol. 12, no. 2, pp. 83-85, Feb. 2005.

[13] R. W. P. King, B. S. Trembly, and J. W. Strohbehn, "The electro magnetic field of an insulated antenna in a conducting of a dielectric medium," IEEE Trans. Microw. Theory Tech., vol. MTT-31, pp. 574-583, Jul. 1983
[14] J. P. Casey and R. Bansal, "The near field of an insulated dipole in 3 dissipative dielectric medium," IEEE Trans. Microw. Theory Tech., vol. 34, no. 4, pp. 459-463, Apr. 1986.

[15] W. L. Stutzman and G. A. Thiele, Antenna Theory and Design. New York: Wiley, 1998, pp. 435-436.

[16] L. L. Tsai, "A numerical solution for the near and far fields of an annular ring of magnetic current," IEEE Trans. Antennas Propag., vol. AP-20, no. 5, pp. 569-576, Sep. 1972.

[17] A. J. Fenn, G. L. Wolf, and R. M. Fogel, "An adaptive microwave phased array for targeted heating of deep tumors in intact breast: animal study results," Int. J. Hypertherm., vol. 15, no. 1, pp. 45-61, 1999.

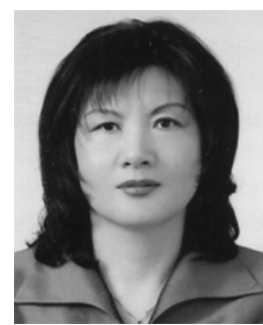

Hee-Ran Ahn (S'90-M'95-SM'99) received the B.S., M.S., and Ph.D. degrees in electronic engineering from Sogang University, Seoul, Korea, in 1988, 1990, and 1994, respectively.

From 1991 to 1995, she was Part-Time Lecturer at Sogang University and Postdoctorial Fellow from 1996 to 1997 at Duisburg-Essen University, Duisburg, Germany. From February 1997 to 2002, she was with the Department of Electrical Engineering at Duisburg-Essen university, Duisburg, Germany, where she worked for the Habilitation dealing with asymmetric passive components in microwave circuits. From 2003 to 2005, she was with the Department of Electrical Engineering and Computer Science at the Korea Advanced Institute of Science and Technology (KAIST), Daejeon, Korea as a Visiting Professor. Since July 2005, she is with the Department of Electronics and Electrical Engineering, Pohang University of Science and Technology (POSTECH), Pohang, Korea. Her interests include high-frequency and microwave circuit design and biomedical application of microwave theory and techniques.

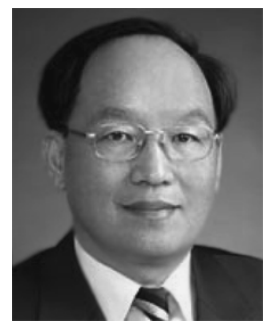

Kwyro Lee (M'80-SM'90) received the B.S. degree in electronics engineering from Seoul National University, Seoul, Korea, in 1976, and the M.S. and Ph.D. degrees from the University of Minnesota, Minneapolis, in 1981 and 1983, respectively, where he did many pioneering works for characterization and modeling of $\mathrm{AlGaAs} / \mathrm{GaAs}$ hetero-junction filed-effect transistor

He was an Engineering General Manager at GoldStar Semi-conductor, Inc., Korea, from 1983 to 1986 for development of the first poly-silicon CMOS products in Korea. He joined Korea Advanced Institute of Science and Technology in 1987 as an assistant professor in Department of Electrical Engineering, where he is now Professor. He has written more than 150 publications in major international journals and conferences. He is the principal author of the book of Semiconductor Device Modeling for VLSI (Englewood Cliffs, NJ: Prentice-Hall, 1993) and one of the developers of AIM-Spice, the world's first SPICE run under windows. Since 1997, he has been the Director of Micro Information and Communication Remote Object-Oriented Systems (MICROS), an Engineering Center of Excellence supported by Korea Science and Engineering Foundation In 1998-2000, he served as the KAIST Dean of Research Affairs as well as the Dean of Institute Development and Cooperation.

Dr. Lee is Life Member of IEEK. During 1990-1996, he served as the Conference Co-chair of Int. Semiconductor Device Research Symposium, Charlottesville, VA. In 1998-2000, the same periods, he also served as the Chairman of IEEE Korea Electron Devices Chapter, and is currently serving as the elected member of AdCom. 